\title{
Strict Fejér Monotonicity by Superiorization of Feasibility-Seeking Projection Methods
}

\author{
Yair Censor* • Alexander J. Zaslavski ${ }^{\dagger}$ \\ Communicated by Jonathan Michael Borwein
}

February 23, 2014. Revised April 19, 2014. Revised: May 26, 2014.

\begin{abstract}
We consider the superiorization methodology, which can be thought of as lying between feasibility-seeking and constrained minimization. It is not quite trying to solve the full fledged constrained minimization problem; rather, the task is to find a feasible point which is superior (with respect to the objective function value) to one returned by a feasibility-seeking only algorithm.

Our main result reveals new information about the mathematical behavior of the superiorization methodology. We deal with a constrained minimization problem with a feasible region, which is the intersection of finitely many closed convex constraint sets, and use the dynamic string-averaging projection method, with variable strings and variable weights, as a feasibility-seeking algorithm. We show that any sequence, generated by the superiorized version of a dynamic stringaveraging projection algorithm, not only converges to a feasible point but, additionally, either its limit point solves the constrained minimization problem or the sequence is strictly Fejér monotone with respect to a subset of the solution set of the original problem.
\end{abstract}

Keywords Bounded perturbation resilience, constrained minimization, convex feasibility problem, dynamic string-averaging projections, strict Fejér monotonicity, subgradients, superiorization methodology, superiorized version of an algorithm.

2010 Mathematics Subject Classification (MSC) 90C25, 90C30, 90C45, 65K10.

*Y. Censor (corresponding author) Department of Mathematics, University of Haifa, Mt. Carmel, Haifa 3498838, Israel. e-mail: yair@math.haifa.ac.il

$\dagger$ A.J. Zaslavski, Department of Mathematics, The Technion - Israel Institute of Technology, Technion City, Haifa 32000, Israel. e-mail: ajzasl@techunix.technion.ac.il 


\section{Introduction}

What is superiorization. The recently developed superiorization methodology (SM) lies between feasibility-seeking and constrained minimization (CM). It is not quite trying to solve the full fledged CM problem; rather, the task is to find a feasible point of the CM problem, that is superior, not necessarily optimal, with respect to the objective function value to one returned by a feasibility-seeking only algorithm. Therefore, the SM can be beneficial for CM problems for which an exact algorithm has not yet been discovered, or when existing exact optimization algorithms are very time consuming or require too much computer space for realistic large problems to be run on commonplace computers. In such cases, efficient feasibility-seeking iterative projection methods that provide non-optimal but constraints-compatible solutions, can be turned by the SM into efficient algorithms for superiorization that will be practically useful from the point of view of the underlying objective function.

For the SM to be useful for a CM problem we need to have an efficient feasibilityseeking algorithm that is in some well-defined sense perturbation resilient. Then the SM uses those permitted perturbations in order to steer the superioized version of the original feasibility-seeking algorithm toward points with lesser, not necessarily minimal, objective function values. The advantage is that in this manner one uses essentially not an optimization algorithm but a superiorized-feasibility-seeking algorithm to attack the CM problem. The latter methods are in many cases very efficient; see, e.g., [1], and, therefore, can save time and computing resources as compared with exact optimization algorithms.

Additionally, in many mathematical formulations of significant real-world technological or physical problems the objective function is exogenous to the modeling process which defines the constraints. In such cases the "faith" of the modeler in the usefulness of an objective function for the application at hand is limited and, as a consequence, it is not worthwhile to invest too much resources in trying to reach an exact constrained minimum point. These notions are rigorously explained in the next sections below.

Contribution. Our main result, in Theorem 4.1 below, establishes a mathematical basis for the behavior of the SM when dealing with a CM problem with a feasible region that is the intersection of finitely many closed convex constraint sets, see Case 2.1] in Section 2 below. We use the dynamic string-averaging projection (DSAP) method, with variable strings and variable weights, algorithmic scheme as a feasibility-seeking algorithm, which is indeed bounded perturbations resilient. The bounded perturbations resilience of the DSAP method has been proved in [2] and the practical behavior of the SM was observed in numerous recent works, see references mentioned below. Our contribution here is the mathematical guarantee of the convergence behavior of the superiorized version of the DSAP algorithm.

Theorem 4.1 below says that any sequence, generated by the superiorized version of a DSAP algorithm, given in Algorithm 4.1 below, will not only converge to a feasible 
point of the underlying CM problem, a fact which is due to the bounded perturbations resilience of the DSAP method, but, additionally, that either its limit point will solve the CM problem (11) or that the sequence is strictly Fejér monotone with respect to; i.e., gets strictly closer to the points of, a subset of the solution set of the CM problem according to (29) below.

Related work. This paper is a sequel to a series of recent publications on the SM [2 13], culminating in [14]. The latter contains a detailed description of the SM, its motivation, and an up-to-date review of SM-related previous work, including a reference to [4] in which it all started, although without using yet the terms superiorization and bounded perturbation resilience. [4] was the first to propose this approach and implement it in practice, but its roots go back to [15, 16] where it was shown that if iterates of a nonexpansive operator converge for any initial point, then its inexact iterates with summable errors also converge. More details on related work appear in [14, Section 3] and in [17, Section 1].

Paper structure. The paper is laid out as follows. Section 2 presents the SM. Preliminaries needed for our study are presented in Section 3 , and the superiorized version of the DSAP algorithm is given in Section 4. The proof of our main result that gives a mathematical basis for the SM is presented in Section 5. Conclusions are given in Section 6.

\section{The Superiorization Methodology}

Consider some mathematically-formulated problem, of any kind or sort, and denote it by $T$. The set of solutions, called the solution set of $T$, is denoted by $\operatorname{SOL}(T)$. The superiorization methodology (SM) of [5, 10, 14] is intended for constrained minimization (CM) problems of the form:

$$
\operatorname{minimize}\left\{\phi(x) \mid x \in \Psi_{T}\right\}
$$

where $\phi: \mathbb{R}^{J} \rightarrow \mathbb{R}$ is an objective function and $\Psi_{T} \subseteq \mathbb{R}^{J}$ is the solution set $\Psi_{T}=$ $\operatorname{SOL}(T)$ of a problem $T$. In [4,5] $\operatorname{SOL}(T)$ was assumed to be nonempty and in later works [10,14] this assumption was removed. Here, however, we adhere to $\Psi_{T}=\operatorname{SOL}(T) \neq \emptyset$ throughout this paper.

To proceed with the SM, the problem $T$ can be just about any mathematical or mathematically-formulated problem for which a "good" iterative algorithm for its solution exists which is bounded perturbation resilient, as explained below. Two widelyused cases of such underlying problems and their set $\Psi_{T}$ come to mind, although the general approach is by no means restricted to those.

Case 2.1 The set $\Psi_{T}$ is the solution set of a convex feasibility problem (CFP) of the form: find a vector $x^{*} \in \Psi_{T}:=\cap_{i=1}^{I} C_{i}$, where the sets $C_{i} \subseteq \mathbb{R}^{J}$ are closed and convex 
subsets of the Euclidean space $\mathbb{R}^{J}$; see, e.g., [18 20] or [37, Chapter 5] for results and references on the broad topic of CFPs or consult [1, 3, 21-27]. In such a case, we deal in (1) with a standard CM problem. This is the case analyzed in this paper.

Case 2.2 The set $\Psi_{T}$ is the solution set of another CM problem which serves as the problem $T$, such as,

$$
\operatorname{minimize}\{J(x) \mid x \in \Omega\} \text {, }
$$

in which case we look at

$$
\Psi_{T}:=\left\{x^{*} \in \Omega \mid J\left(x^{*}\right) \leq J(x) \text { for all } x \in \Omega\right\},
$$

assuming that $\Psi_{T}$ is nonempty. This case has been studied in [8], [7] and [28].

In either case, or any other case for the set $\Psi_{T}$, the SM strives not to solve (1) but rather the task is to find a point in $\Psi_{T}$ which is superior, i.e., has a lower, but not necessarily minimal, value of the $\phi$ objective function value, to one returned by an algorithm that solves the original problem $T$ alone.

This is done in the SM by first investigating the bounded perturbation resilience of an available iterative algorithm designed to solve the original problem $T$ and then proactively using such permitted perturbations in order to steer the iterates of such an algorithm toward lower values of the $\phi$ objective function while not loosing the convergence to a point in $\Psi_{T}$. See [5, 10, 14] for details. A review of superiorizationrelated previous work appears in [14, Section 3].

\section{Preliminaries}

Let $X$ be a Hilbert space equipped with an inner product $\langle\cdot, \cdot\rangle$, which induces a complete norm $\|\cdot\|$. For each $x \in X$ and each nonempty set $E \subseteq X$ define

$$
d(x, E):=\inf \{\|x-y\| \mid y \in E\},
$$

and for each $x \in X$ and each $r>0$ define the closed ball around $x$ with radius $r$ by

$$
B(x ; r):=\{y \in X \mid\|x-y\| \leq r\} .
$$

The following proposition and corollary are well-known, see, e.g., [24] or [22].

Proposition 3.1 Let $D$ be a nonempty, closed and convex subset of $X$. Then, for each $x \in X$ there is a unique point $P_{D}(x) \in D$ (called the projection of $x$ onto $D$ ) satisfying

$$
\left\|x-P_{D}(x)\right\|=\inf \{\|x-y\| \mid y \in D\} .
$$


Moreover,

$$
\left\|P_{D}(x)-P_{D}(y)\right\| \leq\|x-y\| \text { for all } x, y \in X,
$$

and for each $x \in X$ and each $z \in D$,

$$
\left\langle z-P_{D}(x), x-P_{D}(x)\right\rangle \leq 0 \text {. }
$$

Corollary 3.1 Assume that $D$ is a nonempty, closed and convex subset of $X$. Then, for each $x \in X$ and each $z \in D$,

$$
\left\|z-P_{D}(x)\right\|^{2}+\left\|x-P_{D}(x)\right\|^{2} \leq\|z-x\|^{2} .
$$

Suppose that $C_{1}, C_{2}, \ldots, C_{m}$ are nonempty, closed and convex subsets of $X$ where $m$ is a natural number. Set

$$
C:=\cap_{i=1}^{m} C_{i}
$$

and assume throughout that $C \neq \emptyset$. For $i=1,2, \ldots, m$, denote $P_{i}:=P_{C_{i}}$. By an index vector, we a mean a vector $t=\left(t_{1}, t_{2}, \ldots, t_{q}\right)$ such that for all $i=1,2, \ldots, q$, $t_{i} \in\{1,2, \ldots, m\}$ and whose length is $\ell(t)=q$. Define the product of the individual projections onto the sets whose indices appear in the index vector $t$ by

$$
P[t]:=P_{t_{q}} \cdots P_{t_{1}}
$$

and call it a string operator.

A finite set $\Omega$ of index vectors is called fit iff for each $i \in\{1,2, \ldots, m\}$, there exists a vector $t \in \Omega$ such that $t_{s}=i$ for some $s \in\{1,2, \ldots, q\}$. For each index vector $t$ the string operator is nonexpansive, since the individual projections are, i.e.,

$$
\|P[t](x)-P[t](y)\| \leq\|x-y\| \text { for all } x, y \in X,
$$

and also

$$
P[t](x)=x \text { for all } x \in C \text {. }
$$

Denote by $\mathcal{M}$ the collection of all pairs $(\Omega, w)$, where $\Omega$ is a finite fit set of index vectors and

$$
w: \Omega \rightarrow] 0, \infty\left[\text { is such that } \sum_{t \in \Omega} w(t)=1 .\right.
$$

A pair $(\Omega, w) \in \mathcal{M}$ and the function $w$ were called in [4] an amalgamator and a fit weight function, respectively. For any $(\Omega, w) \in \mathcal{M}$ define the convex combination of the end-points of all strings defined by members of $\Omega$ by

$$
P_{\Omega, w}(x):=\sum_{t \in \Omega} w(t) P[t](x), x \in X
$$


It is easy to see that

$$
\left\|P_{\Omega, w}(x)-P_{\Omega, w}(y)\right\| \leq\|x-y\| \text { for all } x, y \in X
$$

and

$$
P_{\Omega, w}(x)=x \text { for all } x \in C .
$$

We will make use of the following condition, known in the literature as bounded regularity, see [18], and assume throughout that it holds.

Condition 3.1 For each $\epsilon>0$ and each $M>0$ there exists a positive $\delta=\delta(\epsilon, M)$ such that for each $x \in B(0, M)$ satisfying $d\left(x, C_{i}\right) \leq \delta$, for all $i=1,2, \ldots, m$, the inequality $d(x, C) \leq \epsilon$ holds.

For the proof of the next proposition see, e.g., [2, Proposition 5].

Proposition 3.2 If the space $X$ is finite-dimensional then Condition 3.1 holds.

We choose an arbitrary fixed number $\Delta \in] 0,1 / m[$ and an integer $\bar{q} \geq m$ and denote by $\mathcal{M}_{*} \equiv \mathcal{M}_{*}(\Delta, \bar{q})$ the set of all $(\Omega, w) \in \mathcal{M}$ such that the lengths of the strings are bounded and the weights are all bounded away from zero, namely,

$$
\mathcal{M}_{*}:=\{(\Omega, w) \in \mathcal{M} \mid \ell(t) \leq \bar{q} \text { and } w(t) \geq \Delta \text { for all } t \in \Omega\}
$$

The dynamic string-averaging projection (DSAP) method with variable strings and variable weights is the following algorithm.

\section{Algorithm 3.1 The DSAP method with variable strings and variable weights}

Initialization: select an arbitrary $x^{0} \in X$,

Iterative step: given an iteration vector $x^{k}$ pick a pair $\left(\Omega_{k}, w_{k}\right) \in \mathcal{M}_{*}$ and calculate the next iteration vector $x^{k+1}$ by

$$
x^{k+1}=P_{\Omega_{k}, w_{k}}\left(x^{k}\right) .
$$

The first prototypical string-averaging algorithmic scheme appeared in [29] and subsequent work on various such algorithmic operators includes [13, 17, 30, 36].

If in the DSAP method one uses only a single index vector $t=(1,2, \ldots, m)$ that includes all constraints indices then the fully-sequential Kaczmarz cyclic projection method; see, e.g., [24, p. 220], is obtained, sometimes called the POCS, for Projections Onto Convex Sets, method; see, e.g., [37, Chapter 5]. For linear hyperplanes as constraints sets the latter is equivalent with the, independently discovered, ART, for Algebraic Reconstruction Technique, in image reconstruction from projections, see [27]. If, at the other extreme, one uses exactly $m$ one-dimensional index vectors $t=(i)$, for $i=$ 
$1,2, \ldots, m$, each consisting of exactly one constraint index, then the fully-simultaneous projection method of Cimmino; see, e.g., [18, page 405], is recovered. In-between these "extremes" the DSAP method allows for a large "arsenal" of many specific feasibilityseeking projection algorithms - to all of which the results of this paper will apply.

For the reader's convenience we quote here the definition of bounded perturbations resilience and the bounded perturbations resilience theorem of the DSAP method, see [2] for details. The next definition was originally given in [5, Definition 1] with a finitedimensional Euclidean space $\mathbb{R}^{J}$ instead of the Hilbert space $X$ in the definition below which is taken from [2].

Definition 3.1 Given a problem $T$, an algorithmic operator $\mathcal{A}: X \rightarrow X$ is said to be bounded perturbations resilient iff the following is true: if the sequence $\left\{x^{k}\right\}_{k=0}^{\infty}$, generated by $x^{k+1}=\mathcal{A}\left(x^{k}\right)$, for all $k \geq 0$, converges to a solution of $T$ for all $x^{0} \in X$, then any sequence $\left\{y^{k}\right\}_{k=0}^{\infty}$ of points in $X$ that is generated by $y^{k+1}=\mathcal{A}\left(y^{k}+\beta_{k} v^{k}\right)$, for all $k \geq 0$, also converges to a solution of $T$ provided that, for all $k \geq 0, \beta_{k} v^{k}$ are bounded perturbations, meaning that $\beta_{k} \geq 0$ for all $k \geq 0$ such that $\sum_{k=0}^{\infty} \beta_{k}<\infty$ and such that the sequence $\left\{v^{k}\right\}_{k=0}^{\infty}$ is bounded.

The convergence properties and the, so called, bounded perturbation resilience of this DSAP method were analyzed in [2].

Theorem 3.1 [2, Theorem 12] Let $C_{1}, C_{2}, \ldots, C_{m}$ be nonempty, closed and convex subsets of $X$, where $m$ is a natural number, $C:=\cap_{i=1}^{m} C_{i} \neq \emptyset$, let $\left\{\beta_{k}\right\}_{k=0}^{\infty}$ be a sequence of non-negative numbers such that $\sum_{k=0}^{\infty} \beta_{k}<\infty$, let $\left\{v^{k}\right\}_{k=0}^{\infty} \subset X$ be a norm bounded sequence, let $\left\{\left(\Omega_{k}, w_{k}\right)\right\}_{k=0}^{\infty} \subset \mathcal{M}_{*}$, for all $k \geq 0$, and let $y^{0} \in X$. Then, any sequence $\left\{y^{k}\right\}_{k=0}^{\infty}$, generated by the iterative formula

$$
y^{k+1}=P_{\Omega_{k}, w_{k}}\left(y^{k}+\beta_{k} v^{k}\right),
$$

converges in the norm of $X$ and its limit belongs to $C$.

\section{The Superiorized Version of the Dynamic String- Averaging Projection Algorithm}

The "superiorized version of an algorithm" has evolved and undergone several modifications throughout the publications on the SM, from the initial [4, pseudocode on page 543] through [6,9, 12] until the most recent [14, "Superiorized Version of the Basic Algorithm" in Section 4]. The next algorithm, called "The superiorized version of the DSAP algorithm", is a further modification of the latest [14, "Superiorized Version of the Basic Algorithm" in Section 4] as we explain in Remark 4.1 below. 
Let $C$ be as in (10), let $\phi: X \rightarrow \mathbb{R}$ be a convex continuous function, and consider the set

$$
C_{\min }:=\{x \in C \mid \phi(x) \leq \phi(y) \text { for all } y \in C\},
$$

and assume that $C_{\min } \neq \emptyset$.

Algorithm 4.1 The superiorized version of the DSAP algorithm

(O) Initialization: Let $N$ be a natural number and let $y^{0} \in X$ be an arbitrary userchosen vector.

(1) Iterative step: Given a current vector $y^{k}$ pick an $N_{k} \in\{1,2, \ldots, N\}$ and start an inner loop of calculations as follows:

(1.1) Inner loop initialization: Define $y^{k, 0}=y^{k}$.

(1.2) Inner loop step: Given $y^{k, n}$, as long as $n<N_{k}$ do as follows:

(1.2.1) Pick a $0<\beta_{k, n} \leq 1$ in a way that guarantees that (this can be done; see Remark 4.1 below)

$$
\sum_{k=0}^{\infty} \sum_{n=0}^{N_{k}-1} \beta_{k, n}<\infty .
$$

(1.2.2) Let $\partial \phi\left(y^{k, n}\right)$ be the subgradient set of $\phi$ at $y^{k, n}$ and define $v^{k, n}$ as follows:

$$
v^{k, n}=\left\{\begin{array}{cl}
-\frac{s^{k, n}}{\left\|s^{k, n}\right\|}, & \text { if } 0 \notin \partial \phi\left(y^{k, n}\right), \\
0, & \text { if } 0 \in \partial \phi\left(y^{k, n}\right),
\end{array}\right.
$$

where $s^{k, n} \in \partial \phi\left(y^{k, n}\right)$.

(1.2.3) Calculate

$$
y^{k, n+1}=y^{k, n}+\beta_{k, n} v^{k, n}
$$

and go to (1.2).

(1.3) Exit the inner loop with the vector $y^{k, N_{k}}$

(1.4) Calculate

$$
y^{k+1}=P_{\Omega_{k}, w_{k}}\left(y^{k, N_{k}}\right)
$$

with $\left(\Omega_{k}, w_{k}\right) \in \mathcal{M}_{*}$, and go back to (1).

\section{Remark 4.1}

1. For step (1.2.1) in Algorithm 4.1 assume that we have available a summable sequence $\left\{\eta_{\ell}\right\}_{\ell=0}^{\infty}$ of positive real numbers (for example, $\eta_{\ell}=a^{\ell}$, where $0<a<$ $1)$. Then we can let the algorithm generate, simultaneously with the sequence $\left\{y^{k}\right\}_{k=0}^{\infty}$, a sequence $\left\{\beta_{k, n}\right\}_{k=0}^{\infty}$ as a subsequence of $\left\{\eta_{\ell}\right\}_{\ell=0}^{\infty}$, by choosing $\beta_{k, n}=\eta_{\ell}$ and increasing the index $\ell$ in every pass through step (1.2.1) in the algorithm, resulting in a positive summable sequence $\left\{\beta_{k, n}\right\}_{k=0}^{\infty}$, as required in (22). This is how it was done in 14. "Superiorized Version of the Basic Algorithm" in Section 4]. 
2. There are some differences between the "Superiorized Version of the Basic Algorithm" in Section 4 of [14] and Algorithm 4.1. In 14] it was the case that $N_{k}=N$ for all $k \geq 0$, whereas here we allow the number of times that the inner loop step (1.1) is exercised to vary from iteration to iteration depending on the iteration index $k$.

3. In our Algorithm 4.1 we do not have to check if $\phi\left(y^{k, n+1}\right) \leq \phi\left(y^{k}\right)$ after (24) in step (1.2.3) of the algorithm, as is done in step 14 of the "Superiorized Version of the Basic Algorithm" in Section 4 of [14]. In spite of this saving shortcut we are able to prove the main result for our Algorithm 4.1, as seen in Theorem 4.1] below.

4. Admittedly, our algorithm is related to only Case 2.1 in Section 2, and uses negative subgradients in step (1.2.2) and not general nonascend steps as in step 8 of the "Superiorized Version of the Basic Algorithm" in Section 4 of [14].

5. Finally, as mentioned before, our findings are related to Case 2.1 for the consistent case $C=\cap_{i=1}^{m} C_{i} \neq \emptyset$ and treat bounded perturbations resilience and not the notion of strong perturbation resilience as in [10,14]. This enables us to prove asymptotic convergence results here but also calls for future research to cover the inconsistent case.

6. Note that the DSAP method, covered here, is a versatile algorithmic scheme that includes, as special cases, the fully sequential projections method and the fully simultaneous projections method as "extreme" structures obtained by putting all sets $C_{i}$ into a single string, or by putting each constraint in a separate string, respectively. Consult the references mentioned in Case 2.1 above for further details and relevant references.

We will prove the following theorem as our main result.

Theorem 4.1 Let $\phi: X \rightarrow \mathbb{R}$ be a convex continuous function, and let $C_{*} \subseteq C_{\min }$ be a nonempty subset of $C_{\text {min }}$. Let $\left.\left.r_{0} \in\right] 0,1\right]$ and $\bar{L} \geq 1$ be such that

$$
|\phi(x)-\phi(y)| \leq \bar{L}\|x-y\| \text { for all } x \in C_{*} \text { and all } y \in B\left(x, r_{0}\right) \text {, }
$$

and suppose that

$$
\left\{\left(\Omega_{k}, w_{k}\right)\right\}_{k=0}^{\infty} \subset \mathcal{M}_{*} .
$$

Then, any sequence $\left\{y^{k}\right\}_{k=0}^{\infty}$, generated by Algorithm 4.1, converges in the norm topology of $X$ to $y^{*} \in C$ and exactly one of the following two cases holds:

(a) $y^{*} \in C_{\min }$; 
(b) $y^{*} \notin C_{\text {min }}$ and there exist a natural number $k_{0}$ and a $\left.c_{0} \in\right] 0,1[$ such that for each $x \in C_{*}$ and each integer $k \geq k_{0}$,

$$
\left\|y^{k+1}-x\right\|^{2} \leq\left\|y^{k}-x\right\|^{2}-c_{0} \sum_{n=1}^{N_{k}-1} \beta_{k, n},
$$

showing that $\left\{y^{k}\right\}_{k=0}^{\infty}$ is strictly Fejér-monotone with respect to $C_{*}$, i.e., that

$$
\left\|y^{k+1}-x\right\|^{2}<\left\|y^{k}-x\right\|^{2}, \text { for all } k \geq k_{0},
$$

because $c_{0} \sum_{n=1}^{N_{k}-1} \beta_{k, n}>0$.

This theorem establishes a mathematical basis for the behavior of the SM when dealing with Case 2.1 in Section 2, i.e., $\Psi_{T}$ is the solution set of a CFP as in (10), assuming that $\Psi_{T}=C \neq \emptyset$, and using the DSAP method algorithmic scheme as a feasibility-seeking algorithm which is indeed bounded perturbations resilient. The bounded perturbations resilience of the DSAP method has been proved in [2] and the practical behavior of the SM was observed in numerous recent works, so, we furnish here a mathematical guarantee of the convergence behavior of the superiorized version of the DSAP Algorithm 4.1 .

Theorem 4.1 tells us that any sequence $\left\{y^{k}\right\}_{k=0}^{\infty}$, generated by Algorithm 4.1, will not only converge to a feasible point of the underlying CFP, which is due to the bounded perturbations resilience of the DSAP method, but, additionally, that either its limit point will solve the CM problem (11) with $\Psi_{T}=C \neq \emptyset$, or that the sequence $\left\{y^{k}\right\}_{k=0}^{\infty}$ is strictly Fejér-monotone with respect to a subset $C_{*}$ of the solution set $C_{\min }$ of the CM problem, according to (28).

This strict Fejér-monotonicity of the sequence $\left\{y^{k}\right\}_{k=0}^{\infty}$ does not suffice to guarantee its convergence to a minimum point of (1), even though the sequence does converge to a limit point in $C$, but it says that the superiorized version algorithm retains asymptotic convergence to a feasible point in $C$, and that the so created feasibility-seeking sequence has the additional property of getting strictly closer, without necessarily converging, to a subset of the solution set of minimizers of the CM problem (11). For properties of Fejér-monotone and strictly Fejér-monotone sequences see, e.g., [18, Theorem 2.16], [24, Subsection 3.3] and 38].

Another result that describes the behavior of the superiorized version of a basic algorithm is [14, Theorm 4.2]. It considers the case of strong perturbation resilience of the underlying basic algorithm, contrary to our result that considers bounded perturbations resilience, and is, therefore, valid to the case of consistent feasible sets. The inability to prove that the superiorized version of a basic algorithm reduces the value of the objective function $\phi$, a fact which was repeatedly observed experimentally, made us use the term "heuristic" in [10,11]. In spite of the strict Fejér monotonicity proven here the question of proving mathematically the reduction of the value of the objective function $\phi$ remains. 
Another open question is to formulate some reasonable further conditions that will help distinguish before hand between the two alternatives in Theorem 4.1 for the behavior of the superiorized version of a DSAP algorithm. Published experimental results repeatedly confirm that reduction of the value of the objective function $\phi$ is indeed achieved, without loosing the convergence toward feasibility, see [2 13]. In some of these cases the SM returns a lower value of the objective function $\phi$ than an exact minimization method with which it is compared, e.g., [14, Table 1].

\section{The Main Result: Strict Fejér Monotonicity in the Superiorization Method}

\subsection{Auxiliary results}

We will need the next two lemmas.

Lemma 5.1 Let $x, y \in X$ and $\Delta>0$ and let $\phi: X \rightarrow \mathbb{R}$ be a convex continuous function.

$$
\text { If } \phi(x)-\phi(y)>\Delta \text { and } v \in \partial \phi(x) \text { then }\langle v, y-x\rangle<-\Delta \text {. }
$$

Proof It follows from the subgradient inequality that,

$$
\langle v, y-x\rangle \leq \phi(y)-\phi(x)<-\Delta
$$

thus proving the lemma.

Lemma 5.2 Under the assumptions of Theorem [4.1, let $\left.\bar{x} \in C_{*}, \Delta \in\right] 0, r_{0}$ ], $\alpha \in] 0,1]$, and $x \in X$ satisfy

$$
\phi(x)-\phi(\bar{x})>\Delta,
$$

and assume that $v \in \partial \phi(x)$ and that $(\Omega, w) \in \mathcal{M}_{*}$. Then, $v \neq 0$ and

$$
y:=P_{\Omega, w}\left(x-\alpha\|v\|^{-1} v\right)
$$

satisfies

$$
\|y-\bar{x}\|^{2} \leq\|x-\alpha\| v\left\|^{-1} v-\bar{x}\right\|^{2} \leq\|x-\bar{x}\|^{2}-2 \alpha(4 \bar{L})^{-1} \Delta+\alpha^{2} .
$$

Proof Equations (30) and (32) imply that $v \neq 0$. By (26), for each $z \in B\left(\bar{x}, 4^{-1} \Delta \bar{L}^{-1}\right)$ we have

$$
\phi(z)-\phi(\bar{x}) \leq \bar{L}\|z-\bar{x}\| \leq 4^{-1} \Delta .
$$

Thus, in view of (35), Lemma 5.1, and (32), we have for all $z \in B\left(\bar{x}, 4^{-1} \Delta \bar{L}^{-1}\right)$,

$$
\langle v, z-x\rangle<-(3 / 4) \Delta
$$


which implies that

$$
\left\langle\|v\|^{-1} v, z-x\right\rangle<0
$$

Defining $\bar{z}:=\bar{x}+4^{-1} \bar{L}^{-1} \Delta\|v\|^{-1} v$, we obtain, by (37),

$$
0>\left\langle\|v\|^{-1} v, \bar{z}-x\right\rangle=\left\langle\|v\|^{-1} v, \bar{x}+4^{-1} \bar{L}^{-1} \Delta\|v\|^{-1} v-x\right\rangle,
$$

which, in turn, yields

$$
\left\langle\|v\|^{-1} v, \bar{x}-x\right\rangle<-4^{-1} \bar{L}^{-1} \Delta .
$$

Defining now $u:=x-\alpha\|v\|^{-1} v$, (39) gives rise to

$$
\begin{aligned}
\|u-\bar{x}\|^{2} & =\|x-\alpha\| v\left\|^{-1} v-\bar{x}\right\|^{2} \\
& =\|x-\bar{x}\|^{2}-2\left\langle x-\bar{x}, \alpha\|v\|^{-1} v\right\rangle+\alpha^{2} \\
& \leq\|x-\bar{x}\|^{2}-2 \alpha(4 \bar{L})^{-1} \Delta+\alpha^{2} .
\end{aligned}
$$

This yields, by (33), the definition of $u$, (16), (17), and the assumption that $\bar{x} \in C_{*}$,

$$
\begin{aligned}
\|y-\bar{x}\|^{2} & =\left\|P_{\Omega, w}(u)-\bar{x}\right\|^{2} \\
& \leq\|u-\bar{x}\|^{2} \leq\|x-\bar{x}\|^{2}-2 \alpha(4 \bar{L})^{-1} \Delta+\alpha^{2},
\end{aligned}
$$

which completes the proof of the lemma.

\subsection{Proof of Theorem 4.1}

We are now ready to prove the main result of our paper, Theorem 4.1.

Proof From Algorithm 4.1, a sequence $\left\{y^{k}\right\}_{k=0}^{\infty}$ that it generates has the property that for each integer $k \geq 1$ and each $h \in\left\{1,2, \ldots, N_{k}\right\}$,

$$
\left\|y^{k, h}-y^{k}\right\|=\left\|\sum_{j=1}^{h}\left(y^{k, j}-y^{k, j-1}\right)\right\| \leq \sum_{n=0}^{N_{k}-1}\left\|y^{k, n}-y^{k, n-1}\right\| \leq \sum_{n=0}^{N_{k}-1} \beta_{k, n}
$$

and, in particular,

$$
\left\|y^{k, N_{k}}-y^{k}\right\| \leq \sum_{n=0}^{N_{k}-1} \beta_{k, n}
$$

so that, by (22),

$$
\sum_{k=0}^{\infty}\left\|y^{k, N_{k}}-y^{k}\right\| \leq \sum_{k=0}^{\infty}\left(\sum_{n=0}^{N_{k}-1} \beta_{k, n}\right) .
$$

The bounded perturbation resilience secured by Theorem 3.1, guarantees the convergence of $\left\{y^{k}\right\}_{k=0}^{\infty}$ to a point in $C$, namely, that there exists

$$
y^{*}=\lim _{k \rightarrow \infty} y^{k} \in C
$$


in the norm topology.

Assume that (a) of Theorem 4.1 does not hold, i.e., that $y^{*} \notin C_{\min }$. Then there is a $\left.\Delta_{0} \in\right] 0, r_{0} / 2[$ such that

$$
\phi\left(y^{*}\right)>\phi(x)+4 \Delta_{0}, \text { for all } x \in C_{\min },
$$

and there is a natural number $k_{0}$ such that for all integers $k \geq k_{0}$, by (22),

$$
\sum_{n=0}^{N_{k}-1} \beta_{k, n}<(16 \bar{L})^{-1} \Delta_{0}
$$

This index $k_{0}$ can be chosen so that additionally for all integers $k \geq k_{0}$ and all $\ell \in$ $\left\{0,1, \ldots, N_{k}\right\}$, by (42), (45) and (46),

$$
\phi\left(y^{k, \ell}\right)>\phi(x)+2 \Delta_{0}, \text { for all } x \in C_{\min } .
$$

Next we apply Lemma 5.2 as follows. Pick an $\bar{x} \in C_{*}$, let $k \geq k_{0}$ where $k_{0}$ is as above, and let $n \in\left\{1,2, \ldots, N_{k}\right\}$. Use (27), (24), (47)-(48) and the fact that $0<\beta_{k, n} \leq 1$ in Algorithm 4.1, and apply Lemma 5.2 with

$$
\alpha=\beta_{k, n-1}, \Delta=2 \Delta_{0}, x=y^{k, n-1}, v=v^{k, n-1}, \text { and }(\Omega, w)=\left(\Omega_{k}, w_{k}\right) .
$$

This leads to

$$
\begin{aligned}
\left\|y^{k, n}-\bar{x}\right\|^{2} & \leq\left\|y^{k, n-1}-\bar{x}\right\|^{2}-2 \beta_{k, n-1}(4 \bar{L})^{-1} 2 \Delta_{0}+\beta_{k, n-1}^{2} \\
& \leq\left\|y^{k, n-1}-\bar{x}\right\|^{2}-\beta_{k, n-1}(4 \bar{L})^{-1} \Delta_{0},
\end{aligned}
$$

because $-3 \beta_{k, n-1}(4 \bar{L})^{-1} \Delta_{0}+\beta_{k, n-1}^{2} \leq 0$. So, for $n=N_{k}$, in view (25), we have

$$
\left\|y^{k+1}-\bar{x}\right\| \leq\left\|y^{k, N_{k}}-\bar{x}\right\| .
$$

By $y^{k, 0}=y^{k}$ in Algorithm 4.1, (151) and (52),

$$
\begin{aligned}
\left\|y^{k}-\bar{x}\right\|^{2}-\left\|y^{k+1}-\bar{x}\right\|^{2} & \geq\left\|y^{k, 0}-\bar{x}\right\|^{2}-\left\|y^{k, N_{k}}-\bar{x}\right\|^{2} \\
& =\sum_{n=0}^{N_{k}-1}\left(\left\|y^{k, n-1}-\bar{x}\right\|^{2}-\left\|y^{k, n}-\bar{x}\right\|^{2}\right) \\
& \geq(4 \bar{L})^{-1} \Delta_{0} \sum_{n=0}^{N_{k}-1} \beta_{k, n},
\end{aligned}
$$

and

$$
\left\|y^{k+1}-\bar{x}\right\|^{2} \leq\left\|y^{k}-\bar{x}\right\|^{2}-(4 \bar{L})^{-1} \Delta_{0} \sum_{n=0}^{N_{k}-1} \beta_{k, n},
$$

which completes the proof of Theorem 4.1 . 


\section{Conclusions}

In very general terms, the superiorization methodology works by taking an iterative algorithm, investigating its perturbation resilience, and then using proactively such perturbations in order to "force" the perturbed algorithm to do something useful. The perturbed algorithm is called the "superiorized version" of the original unperturbed algorithm.

If the original algorithm is efficient and useful for what it is designed to do (computationally efficient and useful in terms of the application at hand), and if the perturbations are simple and not expensive to calculate, then the advantage of this method is that, for essentially the computational cost of the original algorithm, we are able to get something more by steering its iterates according to the perturbations.

This is a very general principle, which has been successfully used in some important practical applications and awaits to be implemented and tested in additional fields; see, e.g., the recent papers [8,39], for applications in intensity-modulated radiation therapy and in nondestructive testing. An important case is when (i) the original algorithm is a feasibility-seeking algorithm, or one that strives to find constraint-compatible points for a family of constraints, and (ii) the perturbations that are interlaced into the original algorithm aim at reducing (not necessarily minimizing) a given merit (objective) function. Since its inception in 2007, the superiorization method has developed and evolved and it seems now worthwhile to distinguish between two directions, that nourish from the same general principle.

One is the direction when only bounded perturbation resilience is used and the constraints are assumed to be consistent (nonempty intersection). Then one treats the "superiorized version" of the original unperturbed algorithm actually as a recursion formula that produces an infinite sequence of iterates and convergence questions are meant in their asymptotic nature. This is the framework in which we work in this paper.

The second direction does not assume consistency of the constraints but uses instead a proximity function that "measures" the violation of the constraints. Instead of seeking asymptotic feasibility, it looks at $\varepsilon$-compatibility and uses the notion of strong perturbation resilience. The same core "superiorized version" of the original unperturbed algorithm might be investigated in each of these directions, but the second is the more practical one whereas the first makes only asymptotic statements.

We propose the terms "weak superiorization" and "strong superiorization" as a nomenclature for the first and second directions, respectively.

Acknowledgments We thank Gabor Herman for an enlightening discussion about terminology and we greatly appreciate the constructive comments of two anonymous reviewers which helped us improve the paper. 


\section{References}

1. Censor, Y., Chen, W., Combettes, P.L., Davidi, R., Herman, G.T.: On the effectiveness of projection methods for convex feasibility problems with linear inequality constraints. Comput. Optim. Appl. 51, 1065-1088 (2012)

2. Censor, Y., Zaslavski, A.J.: Convergence and perturbation resilience of dynamic string-averaging projection methods. Comput. Optim. Appl. 54, 65-76 (2013)

3. Bauschke, H.H., Koch, V.R.: Projection methods: swiss army knives for solving feasibility and best approximation problems with halfspaces. In: Reich, S., Zaslavski, A. (eds.) Proceedings of the Workshop "Infinite Products of Operators and Their Applications", Haifa, 2012 (2013). Accepted for publication. http://arxiv.org/abs/1301.4506 https://people.ok.ubc.ca/bauschke/Research/c16.pdf

4. Butnariu, D., Davidi, R., Herman, G.T., Kazantsev, I.G.: Stable convergence behavior under summable perturbations of a class of projection methods for convex feasibility and optimization problems. IEEE J. Sel. Top. Signal Process. 1, 540-547 (2007)

5. Censor, Y., Davidi, R., Herman, G.T.: Perturbation resilience and superiorization of iterative algorithms. Inverse Probl. 26, 065008 (2010)

6. Davidi, R., Herman, G.T., Censor, Y.: Perturbation-resilient block-iterative projection methods with application to image reconstruction from projections. Int. Trans. Oper. Res. 16, 505-524 (2009)

7. Garduño, E., Herman, G.T.: Superiorization of the ML-EM algorithm. IEEE T. Nucl. Sci. 61, 162-172 (2014)

8. Davidi, R., Censor, Y., Schulte, R.W., Geneser, S., Xing, L.: Feasibility-seeking and superiorization algorithms applied to inverse treatment planning in radiation therapy. Contemp. Math., accepted for publication. http://arxiv.org/abs/1402.1310

9. Herman, G.T., Davidi, R.: Image reconstruction from a small number of projections. Inverse Probl. 24, 045011 (2008)

10. Herman, G.T., Garduño, E., Davidi, R., Censor, Y.: Superiorization: an optimization heuristic for medical physics. Med. Phys. 39, 5532-5546 (2012)

11. Jin, W., Censor, Y., Jiang, M.: A heuristic superiorization-like approach to bioluminescence tomography. In: International Federation for Medical and Biological Engineering (IFMBE) Proceedings, vol. 39, pp. 1026-1029 (2012) 
12. Nikazad, T., Davidi, R., Herman, G.: Accelerated perturbation-resilient blockiterative projection methods with application to image reconstruction. Inverse Probl. 28, 035005 (2012)

13. Penfold, S.N., Schulte, R.W., Censor, Y., Rosenfeld, A.B.: Total variation superiorization schemes in proton computed tomography image reconstruction. Medical Physics 37, 5887-5895 (2010)

14. Censor, Y., Davidi, R., Herman, G.T., Schulte, R.W., Tetruashvili, L.: Projected subgradient minimization versus superiorization. J. Optim. Theory Appl. 160, 730747 (2014)

15. Butnariu, D., Reich, S., Zaslavski, A.J.: Convergence to fixed points of inexact orbits of Bregman-monotone and of nonexpansive operators in Banach spaces, in: H.F. Nathansky, H.F., de Buen, B.G., Goebel, K., Kirk, W.A., Sims, B. (eds.) Fixed Point Theory and its Applications, (Conference Proceedings, Guanajuato, Mexico, 2005) Yokahama Publishers, Yokahama, Japan, pp. 11-32, 2006

16. Butnariu, D., Reich, S., Zaslavski, A.J.: Stable convergence theorems for infinite products and powers of nonexpansive mappings, Numer. Funct. Anal. Optim. 29, 304-323 (2008)

17. Censor, Y., Zaslavski, A.J.: String-averaging projected subgradient methods for constrained minimization. Optim. Methods Softw. 29, 658-670 (2014)

18. Bauschke, H.H., Borwein, J.M.: On projection algorithms for solving convex feasibility problems. SIAM Rev. 38, 367-426 (1996)

19. Byrne, C.L.: Applied Iterative Methods. AK Peters, Wellsely, MA, USA (2008)

20. Chinneck, J.W.: Feasibility and Infeasibility in Optimization: Algorithms and Computational Methods. Springer, New York, NY, USA (2007)

21. Aharoni, R., Censor, Y.: Block-iterative projection methods for parallel computation of solutions to convex feasibility problems. Linear Algebra Appl. 120, 165-175 (1989)

22. Bauschke, H.H., Combettes, P.L.: Convex Analysis and Monotone Operator Theory in Hilbert Spaces. Springer, New York, NY, USA (2011)

23. Bauschke, H.H., Matoušková, E., Reich, S.: Projection and proximal point methods: convergence results and counterexamples. Nonlinear Anal-Theor. 56, 715-738 (2004) 
24. Cegielski, A.: Iterative Methods for Fixed Point Problems in Hilbert Spaces. Lecture Notes in Mathematics, vol. 2057. Springer, Berlin (2012)

25. Escalante, R., Raydan, M.: Alternating Projection Methods. Society for Industrial and Applied Mathematics (SIAM), Philadelphia, PA, USA, (2011)

26. Galántai, A.: Projectors and Projection Methods. Kluwer Academic Publishers, Dordrecht, The Netherlands (2004)

27. Herman, G.T.: Fundamentals of Computerized Tomography: Image Reconstruction from Projections, 2nd edn. Springer, Berlin (2009)

28. Luo, S., Zhou, T.: Superiorization of EM algorithm and its application in singlephoton emission computed tomography (SPECT). Inverse Probl. Imaging 8, 223$246(2014)$

29. Censor, Y., Elfving, T., Herman, G.T.: Averaging strings of sequential iterations for convex feasibility problems. In: Butnariu, D., Censor, Y., Reich, S. (eds.) Inherently Parallel Algorithms in Feasibility and Optimization and Their Applications, pp. 101-114. Elsevier, Amsterdam (2001)

30. Censor, Y., Segal, A.: On the string averaging method for sparse common fixed point problems. Int. Trans. Oper. Res. 16, 481-494 (2009)

31. Censor, Y., Segal, A.: On string-averaging for sparse problems and on the split common fixed point problem. Contemp. Math. 513, 125-142 (2010)

32. Censor, Y., Tom, E.: Convergence of string-averaging projection schemes for inconsistent convex feasibility problems. Optim. Methods Softw. 18, 543-554 (2003)

33. Crombez, G.: Finding common fixed points of strict paracontractions by averaging strings of sequential iterations. J. Nonlinear Conv. Anal. 3, 345-351 (2002)

34. Gordon, D., Gordon, R.: Component-averaged row projections: A robust, blockparallel scheme for sparse linear systems. SIAM J. Sci. Comput. 27, 1092-1117 (2005)

35. Penfold, S.N., Schulte, R.W., Censor, Y., Bashkirov, V., McAllister, S., Schubert, K.E., Rosenfeld, A.B.: Block-iterative and string-averaging projection algorithms in proton computed tomography image reconstruction. In: Censor, Y., Jiang, M.,Wang, G. (eds.) Biomedical Mathematics: Promising Directions in Imaging, Therapy Planning and Inverse Problems, pp. 347-367. Medical Physics, Madison, WI, USA (2010) 
36. Rhee, H.: An application of the string averaging method to one-sided best simultaneous approximation. J. Korean Soc. Math. Edu., Series B, Pure and Applied Mathematics 10, 49-56 (2003)

37. Censor, Y., Zenios, S.A.: Parallel Optimization: Theory, Algorithms, and Applications. Oxford University Press, New York, NY, USA (1997)

38. Schott, D.: Basic properties of Fejér monotone sequences. Rostocker Math. Kolloq. $49,57-74(1995)$

39. Schrapp, M.J., Herman, G.T.: Data fusion in X-ray computed tomography using an superiorization approach. Rev. Sci. Instrum. 85, 053701 (2014) 